\title{
Endoscopic graduated multiangle, multicorridor resection of juvenile nasopharyngeal angiofibroma: an individualized, tailored, multicorridor skull base approach
}

\author{
James K. Liu, MD, ${ }^{1-3}$ Qasim Husain, MD, ${ }^{2}$ Vivek Kanumuri, MD, ${ }^{2}$ Mohemmed N. Khan, MD, ${ }^{2}$ \\ Zachary S. Mendelson, BS, ${ }^{1}$ and Jean Anderson Eloy, MD1-3 \\ Departments of ${ }^{1}$ Neurological Surgery and ${ }^{2}$ Otolaryngology-Head Neck Surgery, and ${ }^{3}$ Center for Skull Base and Pituitary \\ Surgery, Neurological Institute of New Jersey, Rutgers New Jersey Medical School, Newark, New Jersey
}

\begin{abstract}
OBJECTIVE Juvenile nasopharyngeal angiofibromas (JNAs) are formidable tumors because of their hypervascularity and difficult location in the skull base. Traditional transfacial procedures do not always afford optimal visualization and illumination, resulting in significant morbidity and poor cosmesis. The advent of endoscopic procedures has allowed for resection of JNAs with greater surgical freedom and decreased incidence of facial deformity and scarring.

METHODS This report describes a graduated multiangle, multicorridor, endoscopic approach to JNAs that is illustrated in 4 patients, each with a different tumor location and extent. Four different surgical corridors in varying combinations were used to resect JNAs, based on tumor size and location, including an ipsilateral endonasal approach (uninostril); a contralateral, transseptal approach (binostril); a sublabial, transmaxillary Caldwell-Luc approach; and an orbitozygomatic, extradural, transcavernous, infratemporal fossa approach (transcranial). One patient underwent resection via an ipsilateral endonasal uninostril approach (Corridor 1) only. One patient underwent a binostril approach that included an additional contralateral transseptal approach (Corridors 1 and 2). One patient underwent a binostril approach with an additional sublabial Caldwell-Luc approach for lateral extension in the infratemporal fossa (Corridors 1-3). One patient underwent a combined transcranial and endoscopic endonasal/sublabial Caldwell-Luc approach (Corridors 1-4) for an extensive JNA involving both the lateral infratemporal fossa and cavernous sinus.
\end{abstract}

RESULTS A graduated multiangle, multicorridor approach was used in a stepwise fashion to allow for maximal surgical exposure and maneuverability for resection of JNAs. Gross-total resection was achieved in all 4 patients. One patient had a postoperative CSF leak that was successfully repaired endoscopically. One patient had a delayed local recurrence that was successfully resected endoscopically. There were no vascular complications.

CONCLUSIONS An individualized, multiangle, multicorridor approach allows for safe and effective surgical customization of access for resection of JNAs depending on the size and exact location of the tumor. Combining the endoscopic endonasal approach with a transcranial approach via an orbitozygomatic, extradural, transcavernous approach may be considered in giant extensive JNAs that have intracranial extension and intimate involvement of the cavernous sinus.

http://thejns.org/doi/abs/10.3171/2014.12.JNS141696

KEY WORDS juvenile nasopharyngeal angiofibroma; sinonasal tumor; anterior skull base tumor; endoscopic anterior skull base tumor resection; infratemporal fossa; vascular sinonasal tumor; oncology

$\mathrm{J}$ UVENILE nasopharyngeal angiofibromas (JNAs) are rare, benign, highly vascular, and nonencapsulated tumors that account for up to $0.05-0.5 \%$ of all head and neck tumors. ${ }^{13}$ They are composed of an irregular network of blood vessels set in fibroblastic stroma. ${ }^{3}$ The most common presentation of this tumor includes painless nasal obstruction, recurrent unilateral epistaxis, and a na- sopharyngeal mass in an adolescent male patient. ${ }^{31}$ These tumors tend to be locally aggressive, spreading from the nasopharynx anteriorly into the nasal cavity, laterally into the pterygopalatine fossa (PPF), and superiorly into the intracranial cavity. ${ }^{21,25,28}$ The vascular nature of these tumors has been associated with life-threatening epistaxis and hemorrhage..$^{15}$ 
Diagnostic workup for JNA includes flexible endoscopic examination, CT scanning, and MRI. CT imaging is best used for determining bony changes, whereas MRI looks for soft-tissue destruction. ${ }^{19}$ Anterior bowing of the posterior maxillary wall, the Holman-Miller sign, is pathognomonic for JNA. ${ }^{37}$ A variety of staging criteria systems are used, $,, 6,11,23,27,32,33$ each based on tumor spread. In addition, angiography is often performed to identify the primary vessels that feed the tumor, and allows for preoperative embolization to reduce intraoperative blood loss. ${ }^{21}$

The treatment of choice for JNA is surgical excision, either by endoscopic, endoscopic-assisted, or open surgical approaches..$^{16,26,28}$ With the advent of minimally invasive endoscopic techniques, however, there have been several studies assessing the effectiveness of endoscopic resection of JNA.4,7,12,18,20,22,24,30,35,36 In particular, the infratemporal fossa (ITF) is one of the most difficult areas to access during skull base surgery. More extensive JNAs that extend intracranially or extend into the lateral regions of the ITF become more challenging to resect via a purely endoscopic endonasal approach. Combined approaches that incorporate a transcranial approach and/or a sublabial Caldwell-Luc maxillotomy may be necessary in some cases when a purely endoscopic endonasal approach reaches its surgical limitations. Although visualization of the lateral ITF can be achieved with angled endoscopes, the surgical maneuverability of instruments via a binostril approach is limited by the nasal pyriform aperture.

We have previously reported on a cadaveric study illustrating a graduated multiangle endoscopic approach to the ITF that uses multiple operative corridors that complement the binostril endoscopic corridors to achieve adequate access and surgical freedom to lesions of the ITF. ${ }^{8}$ In this paper, we apply the principles of multicorridor surgery and describe a graduated multiangle, multicorridor approach for resection of complex JNAs. Four different surgical corridors (ipsilateral nostril, contralateral nostril, sublabial Caldwell-Luc maxillotomy, and transcranial) were used in various combinations in a stepwise fashion depending on the size and location of the JNA. We illustrate this concept in 4 patients with JNAs of various sizes and tumor extension to demonstrate how graduated use of multiple surgical corridors provides individualized access to JNAs involving deep areas of the skull base, to facilitate effective oncologic resection.

\section{Case Illustrations and Surgical Approaches}

Four patients with JNAs from the PPF and/or ITF, each with a unique extent and location in the skull base, are pre- sented to illustrate the concept of a graduated multiangle, multicorridor surgical approach. Each of these patients underwent resection via this approach using a combination of the following 4 surgical corridors, based on tumor size, location, and extent (Table 1): 1) an ipsilateral endonasal approach (uninostril); 2) a contralateral transseptal approach (binostril); 3) a sublabial, endoscopic, Caldwell-Luc maxillotomy approach; and 4) an orbitozygomatic, extradural, transcavernous, microsurgical approach (transcranial). One patient underwent resection via an ipsilateral endonasal uninostril approach (Corridor 1) only. One patient underwent a binostril approach that included an additional contralateral, transseptal, binostril approach (Corridors 1 and 2). One patient underwent a binostril approach with an additional sublabial Caldwell-Luc approach for lateral extension into the ITF (Corridors 1-3). One patient underwent a combined transcranial and endoscopic endonasal/ sublabial Caldwell-Luc approach (Corridors 1-4) for an extensive JNA tumor involving both the lateral ITF and cavernous sinus. The clinical reports for each of these 4 cases are detailed. This study qualifies as human subject research, and the protocol was approved by the institutional review board of the Rutgers University, New Jersey Medical School. The various approaches used were deemed feasible and safe based on previous cadaveric studies. ${ }^{8}$

\section{Case 1: Ipsilateral Endoscopic Endonasal Approach (Uninostril Corridor)}

A 22-year-old man with a history of worsening leftsided nasal obstruction presented with recurrent epistaxis. Nasal endoscopy revealed a smooth-walled mass obstructing the left nasal cavity between the middle turbinate and the nasal septum. CT and MR images of the paranasal sinuses revealed a contrast-enhanced lesion between the left middle turbinate and nasal septum measuring approximately $4.7 \times 4.4 \times 3.2 \mathrm{~cm}$ and extending posteriorly to the nasopharynx, left PPF, and through the sphenoid rostrum on the left side. No ITF extension was noted (Fig. 1).

A left, endoscopic, ipsilateral, endonasal transpterygoid approach was used to access this tumor via a uninostril corridor. A modified left medial maxillectomy was performed to provide access to the PPF in the event a sphenopalatine or internal maxillary artery ligation was necessary. The middle turbinate was then removed for better visualization and access to the mass. This allowed for full exposure of the lesion and a successful complete circumferential resection of the mass was achieved. Hemostasis was maintained throughout the procedure (Fig. 2).

Postoperatively, the patient did well and did not have any major complaints. He was discharged to home on

TABLE 1. Multicorridor approach for JNA based on tumor location

\begin{tabular}{cccll}
\hline Case No. & Age $(\mathrm{yrs})$ & Sex & \multicolumn{1}{c}{ Tumor Location } & Surgical Corridor(s) Used \\
\hline 1 & 22 & M & Lt NC, Lt PPF & Uninostril \\
\hline 2 & 22 & M & Bilat NC, Lt PPF, Lt medial ITF & Binostril \\
\hline 3 & 13 & M & Bilat NC, Rt PPF, Rt lateral ITF, Rt IOF & Binostril + Caldwell-Luc \\
\hline 4 & 17 & M & Lt NC, L PPF, Lt lateral ITF, Lt SOF, IC, CS & Binostril + Caldwell-Luc + transcranial \\
\hline
\end{tabular}

CS = cavernous; $I C$ = intracranial; IOF = inferior orbital fissure; NC = nasal cavity; SOF = superior orbital fissure. 

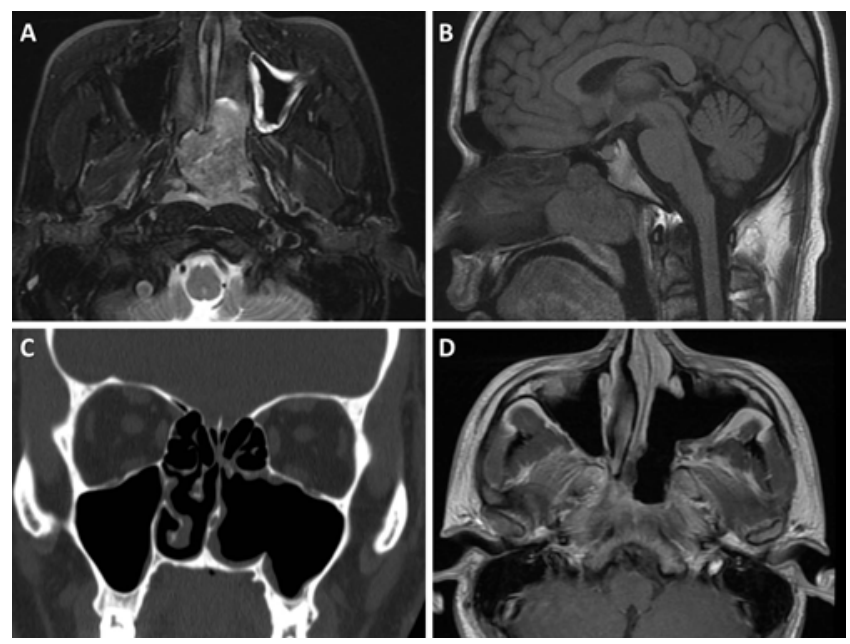

FIG. 1. Case 1. A and B: Preoperative T2-weighted axial (A) and T1-weighted sagittal (B) MR images showing a JNA in the nasal cavity and left PPF. A left uninostril, endoscopic endonasal approach was performed. C and D: Postoperative coronal CT scan (C) and axial T1weighted Gd-enhanced MR image (D) demonstrating complete resection of tumor without residual or recurrent lesion.

postoperative Day 2. Six months postoperatively, he was doing well, with no evidence of recurrence and no further episodes of epistaxis.

\section{Case 2: Ipsilateral Endoscopic Endonasal Approach and Contralateral Endonasal Septotomy (Binostril Corridor)}

A 22-year-old man presented with new-onset seizures and a history of epistaxis. CT and MRI demonstrated a 5.6 $\times 3.6 \times 6.1-\mathrm{cm}$ heterogeneously enhanced soft-tissue mass of the left PPF. The mass extended medially into the nasopharynx and paraclival region, anteriorly into bilateral nasal cavities, and superiorly to the sphenoid sinuses and left posterior ethmoid air cells. It extended through the left PPF into the medial ITF. There was thinning of the floor of the middle cranial fossa without invasion (Fig. 3).

Embolization of the tumor was performed preoperatively. A binostril, endoscopic endonasal transpterygoid approach was performed to resect this lesion (Video 1).

VIDEO 1. Intraoperative video of Case 2 demonstrating a binostril endoscopic endonasal approach for resection of a JNA. Copyright James K. Liu. Published with permission. Click here to view.

Initially, an endoscopic ipsilateral, modified medial maxillectomy was performed to gain wide access to the maxillary sinus and lateral portions of the tumor. A transnasal septotomy was also performed by raising bilateral mucoperichondrial/mucoperiosteal flaps, which were pushed posteriorly into the nasopharynx to protect them during the procedure. A window was then made in the nasal septum by resecting necessary bone and cartilage while preserving an adequate dorsal and caudal strut. This allowed for improved visualization and instrumentation laterally by providing a wider angle of attack through the contralateral nasal cavity (Fig. 4). It also permitted the use of a 2-surgeon, 3- to 4-handed surgical technique through 2 nostril working corridors. A septotomy was preferred over a posterior septectomy because it allowed for a more
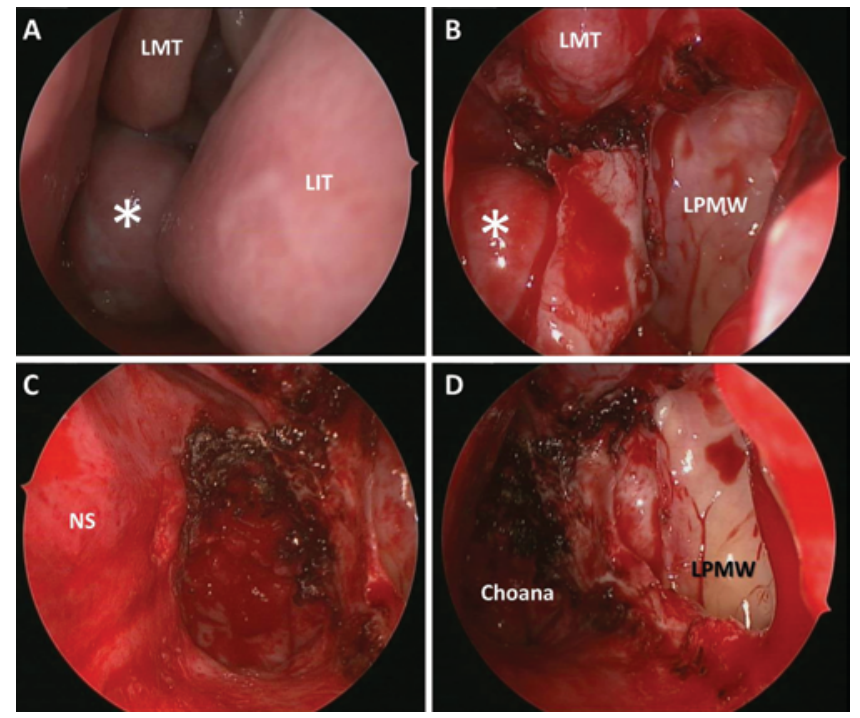

FIG. 2. Case 1. Intraoperative endoscopic views provided by a $30^{\circ}$ angled endoscope. A: The tumor (asterisk) is located in the posterior nasopharynx below the left middle turbinate (LMT) and posterior to the left inferior turbinate (LIT). B: A left uninostril approach with an endoscopic medial maxillectomy was performed to better expose the lateral aspect of the tumor. C and D: Angled endoscopic views of the medial (C) and lateral (D) aspects of the resection cavity after complete tumor removal. LIT = left inferior turbinate; LMT = left middle turbinate; LPMW = left posterior maxillary wall; NS = nasal septum. Figure is available in color online only.

anterior opening in the septum, which provided a better angle to the medial portions of the ITF without requiring an additional Caldwell-Luc maxillotomy approach. A gross-total resection was achieved (Fig. 3); however, the bone covering the temporal lobe dura had been made very thin by the tumor and a small dural defect with slow CSF egress (low-flow leak) was identified. This was repaired with 2 layers of a regenerative tissue matrix (Alloderm; LifeCell), one as an underlay and another as an overlay. The 2 mucoperichondrial/mucoperiosteal flaps were reapproximated anteriorly to cover the septal defect.

Postoperatively, the patient did well and was discharged home on postoperative Day 4 without any CSF leakage. Four months postoperatively, the skull base defect was well covered and the nasal septum had healed without perforation.

\section{Case 3: Ipsilateral Endoscopic Endonasal Approach, Contralateral Endonasal Septotomy, and Ipsilateral Endoscopic Caldwell-Luc Maxillotomy (Binostril and Transmaxillary Corridors)}

A 13-year-old boy initially presented with nasal obstruction. Nasal endoscopy showed a nasopharyngeal mass extending into the right nasal cavity. CT and MRI revealed a $3.7 \times 5.0 \times 4.5-\mathrm{cm}$ vascular mass involving the nasopharynx extending into bilateral nasal cavities, bilateral sphenoid sinuses, and the right PPF and ITF. There was evidence of extension into the right inferior orbital fissure and erosion of the anterior clivus and pterygoid plate (Fig. 5). 


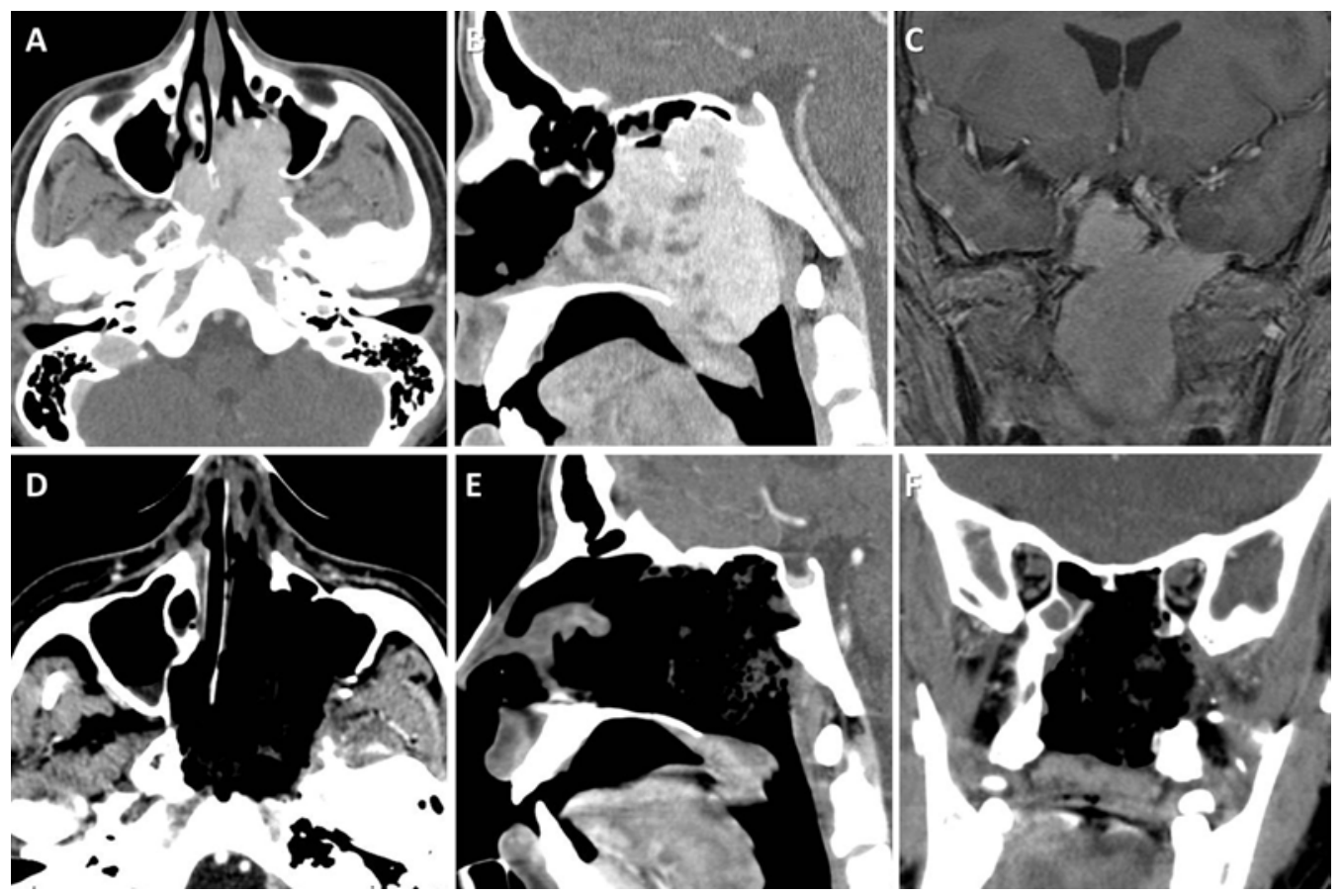

FIG. 3. Case 2. A-C: Preoperative images (axial postcontrast CT scan [A], sagittal postcontrast CT scan [B], and T1-weighted post-Gd coronal MR image [C]) showing a JNA involving bilateral nasal cavity and left PPF with lateral extension into the ITF. A binostril endoscopic, endonasal, transpterygoid approach was performed to achieve complete resection of the tumor. D-F: Postoperative postcontrast CT scans (axial [D], sagittal [E], and coronal [F]) demonstrating no evidence of residual tumor.
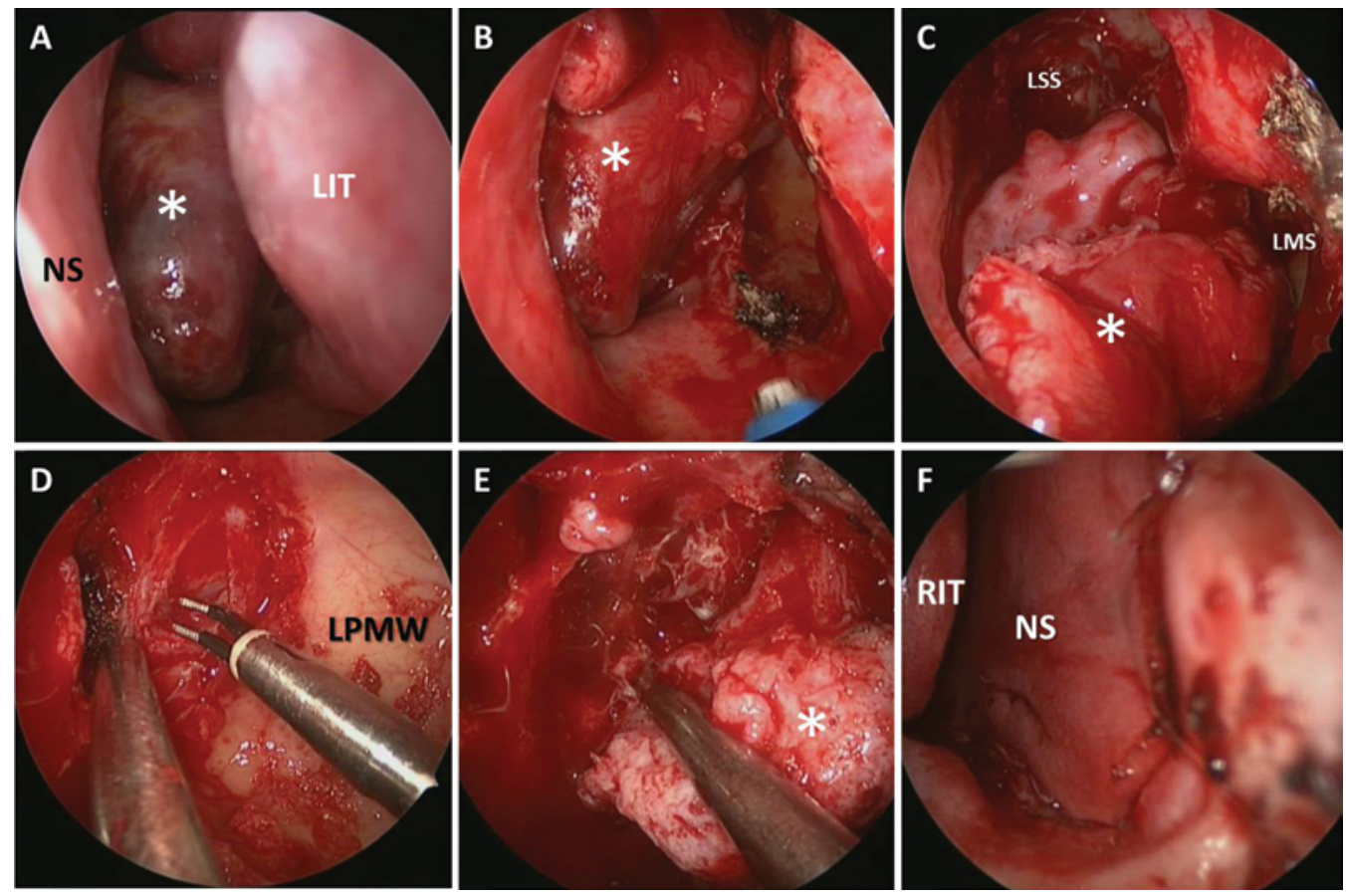

FIG. 4. Case 2. Intraoperative endoscopic views of the tumor. A-C: Endoscopic view of the mobilized tumor (asterisk) through left nasal cavity after a modified left medial maxillectomy. D and E: A $30^{\circ}$-angled endoscopic view from contralateral right nasal cavity through the septotomy window, showing a bipolar instrument in the left nasal cavity during sphenopalatine artery cauterization and initial tumor resection. The right septotomy flap was replaced and sutured. LMS = left maxillary sinus; LSS = left sphenoid sinus; RIT = right inferior turbinate. See Fig. 2 for definitions of other abbreviations. Figure is available in color online only. 

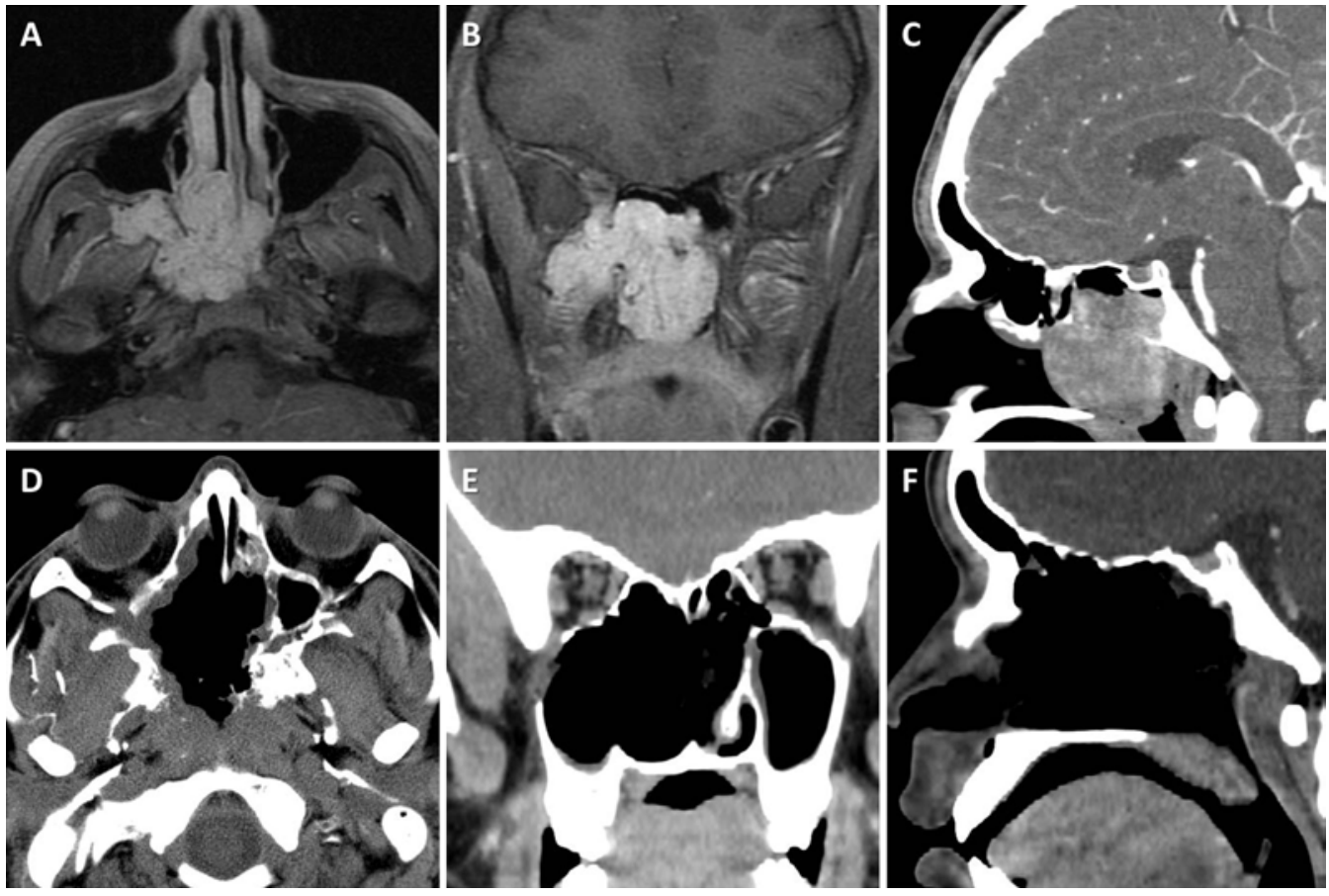

FIG. 5. Case 3. A-C: Preoperative imaging (axial T1-weighted post-Gd MR image [A], coronal T1-weighted post-Gd MR image [B], and postcontrast sagittal CT scan [C]) showing a JNA involving bilateral nasal cavity, right PPF, and far-lateral ITF. A 3-corridor approach using a binostril endoscopic endonasal approach and right sublabial Caldwell-Luc maxillotomy was performed to completely remove the tumor. D-F: Postoperative enhanced CT scans (axial [D], coronal [E], and sagittal [F]) show complete tumor removal without evidence of residual tumor.

A combined endoscopic, multiangle, binostril, endoscopic endonasal, transpterygoid, and sublabial CaldwellLuc maxillotomy approach was used to access the tumor after preoperative angioembolization. A posterior septectomy and an ipsilateral medial maxillectomy were performed. This allowed for wide exposure and a wide-angle approach to the ITF. The additional Caldwell-Luc maxillotomy provided a third corridor for direct access to the far-lateral portions of the ITF that were difficult to reach with the binostril corridors (Fig. 6). The endoscope and surgical instruments were placed interchangeably in all of the 3 surgical corridors in various combinations to provide the proper visualization and surgical freedom to achieve a gross-total resection of this extensive tumor.

Postoperatively, the patient was neurologically intact without complications. He was discharged to home on postoperative Day 4. The patient later developed geometrical alopecia from the radiation exposure associated with angioembolization. Six months postoperatively, he was doing well without any evidence of tumor recurrence, and had total resolution of the alopecia (Fig. 5).

\section{Case 4: Ipsilateral Endoscopic Endonasal Approach, Contralateral Endonasal Septotomy, Ipsilateral Endoscopic Caldwell-Luc Maxillotomy, and Ipsilateral Orbitozygomatic Extradural Transcavernous Approach (Binostril-Transmaxillary-Transcranial Corridors)}

A 17-year-old male patient presented with recurrent epistaxis. Nasal endoscopy revealed a mass obstructing the left nasal cavity and deviating the septum to the right.
Imaging demonstrated a $5 \times 7 \times 6.5-\mathrm{cm}$ highly vascular tumor centered in the left PPF. It extended medially into the nasal cavity and nasopharynx, and laterally into the masticator and buccal spaces and lateral ITF region. The foramen rotundum was widened with tumor extending intracranially into the cavernous sinus. There was evidence of invasion of the orbital floor with preservation of the intraconal space and extension of tumor to the epidural space of the left temporal lobe through the left superior orbital fissure (Fig. 7).

A multicorridor, multiangle approach (4 corridors) was performed using a combined single-stage, binostril, endoscopic endonasal Caldwell-Luc and orbitozygomatic transcavernous approach after preoperative embolization of the tumor (Fig. 8, Video 2).

VIDEO 2. Intraoperative video of Case 4 demonstrating a multiangle, multicorridor resection of a complex JNA using a combined strategy comprising a binostril endoscopic endonasal approach,

Caldwell-Luc maxillotomy, and an orbitozygomatic transcavernous approach. Copyright James K. Liu. Published with permission. Click here to view.

Because of intracranial extension and involvement of the cavernous sinus, a decision was made to perform an orbitozygomatic extradural transcavernous approach to decompress and dissect the tumor off of the cavernous sinus and cavernous carotid artery, and remove the portion of the tumor that had been encroaching into this space (Fig. 8). Once this was achieved, the remaining extracranial portion of the tumor was approached using the 3 endoscopic portals from below (combined binostril and sub- 

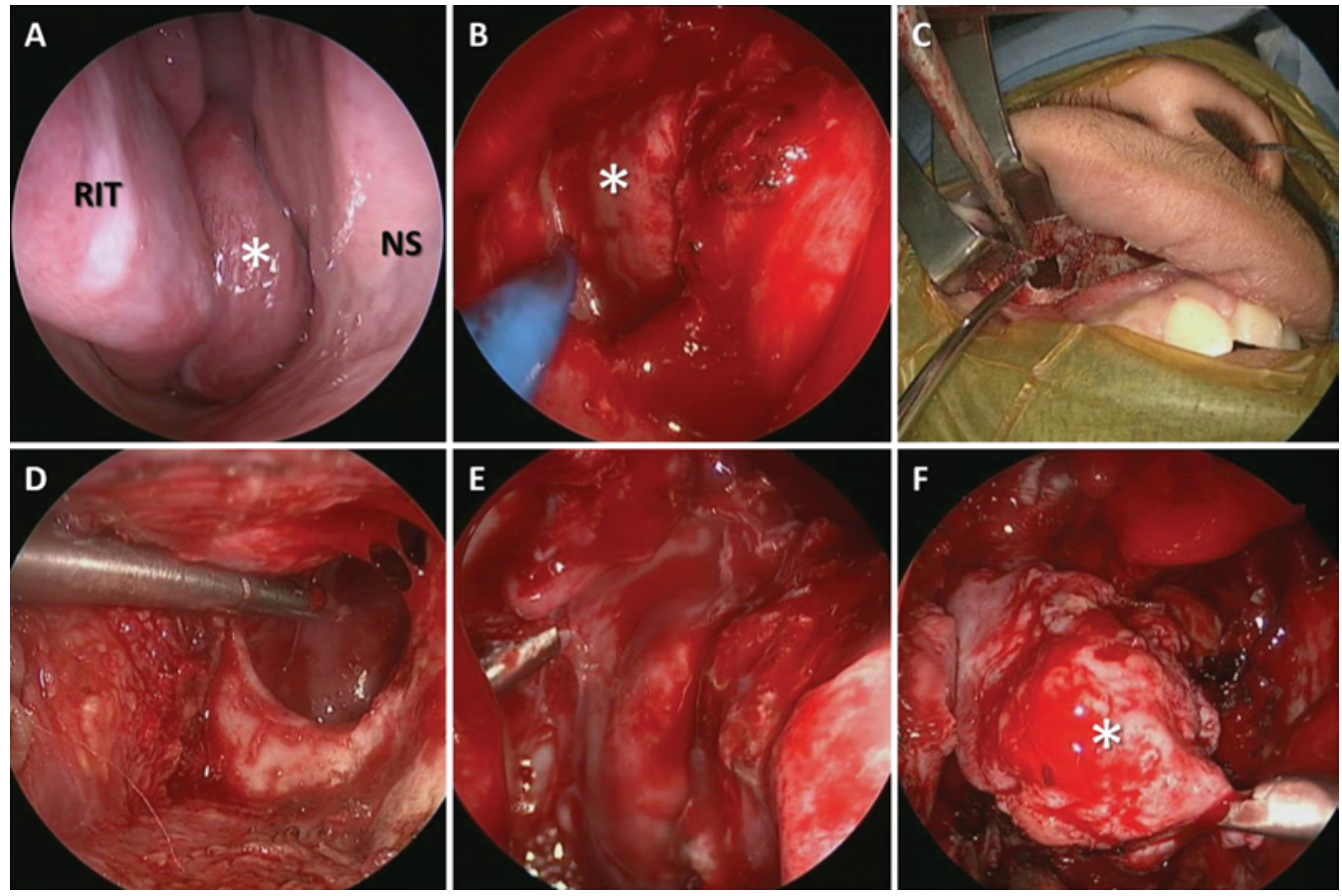

FIG. 6. Case 3. Intraoperative endoscopic views. A: The tumor (asterisk) is seen in the right nasal cavity. B: A left modified medial maxillectomy view of the tumor (asterisk) in the ITF. C: Initial endoscopic sublabial Caldwell-Luc maxillotomy approach. D: Endoscopic view of the tumor from the Caldwell-Luc approach. E: Close-up endonasal view through the Caldwell-Luc opening, showing the lateral extent of the tumor in the ITF. F: Tumor extirpation through the contralateral left nasal cavity. See Figs. 2 and 4 for definition of abbreviations. Figure is available in color online only.

labial Caldwell-Luc maxillotomy) to resect the tumor in the nasal cavity, PPF, and ITF (Fig. 9). The Caldwell-Luc corridor provided excellent access and visualization of the tumor extending laterally into the ITF and buccal space.

The Caldwell-Luc approach was performed by making an ipsilateral sublabial incision and elevating the soft tissue and periosteum off the anterior maxillary sinus wall. An anterior maxillary sinus antrostomy was then performed, which allowed for better visualization and access to the tumor in the far lateral portions of the ITF. An endoscopic, modified medial maxillectomy was then performed on the ipsilateral side for wide exposure and improved endonasal access to the PPF and ITF. Finally, a posterior septectomy window was then created to allow for a binostril 3- to 4-handed technique. All portions of the tumor could now be accessed both for visualization and instrumentation maneuverability through a combination of these 4 corridors (Fig. 9). A gross-total resection was achieved with preservation of V1, V2, and V3.

Postoperatively, the patient was neurologically intact with the exception of mild V2 numbness, which improved at 1-year follow-up. On postoperative Day 2, the patient manifested a CSF leak arising from the temporal fossa defect. This was successfully reconstructed endoscopically with a multilayered acellular dermal allograft repair. Immediate postoperative MRI on Day 1 and at 4 months showed complete resection of the tumor without any evidence of residual or recurrent tumor (Fig. 10).

Six months postoperatively, a surveillance contrastenhanced CT scan revealed a small recurrent lesion in the pterygoid and preclival region. The patient underwent successful binostril endoscopic endonasal resection of the tumor without complications. At 33 months' follow-up, the patient remained neurologically intact without tumor recurrence.

\section{Discussion}

JNA is a rare entity with an incidence reported to be approximately 0.4 cases per million, and it predominantly affects adolescent boys. ${ }^{13}$ These tumors typically present with epistaxis, nasal obstruction, and a nasopharyngeal mass. ${ }^{14} \mathrm{CT}$ and MRI are predominantly used to determine localization and staging for JNAs, which are typically found in the nose and PPF, with bony erosion seen on CT scanning. ${ }^{19}$ However, these tumors can grow into adjacent compartments of the skull base, such as the ITF and intracranial cavity. JNA that involves multiple compartments can be challenging to access surgically and has previously been approached by external open transfacial procedures that can carry substantial morbidity.10,12,26,38

With the introduction of endoscopic skull base techniques, the surgical landscape has changed to now include endoscopic approaches as well as endoscope-assisted approaches to provide access to these tumors. ${ }^{4,7,9,12,18,20,30,36}$ With this shift in surgical paradigms, the staging criteria used for JNA were revised by Snyderman et al. ${ }^{33}$ to place a higher emphasis on cranial base extension and remaining vascularity postembolization, characteristics they believed to be more predictive of morbidity and tumor recur- 
J. K. Liu et al.
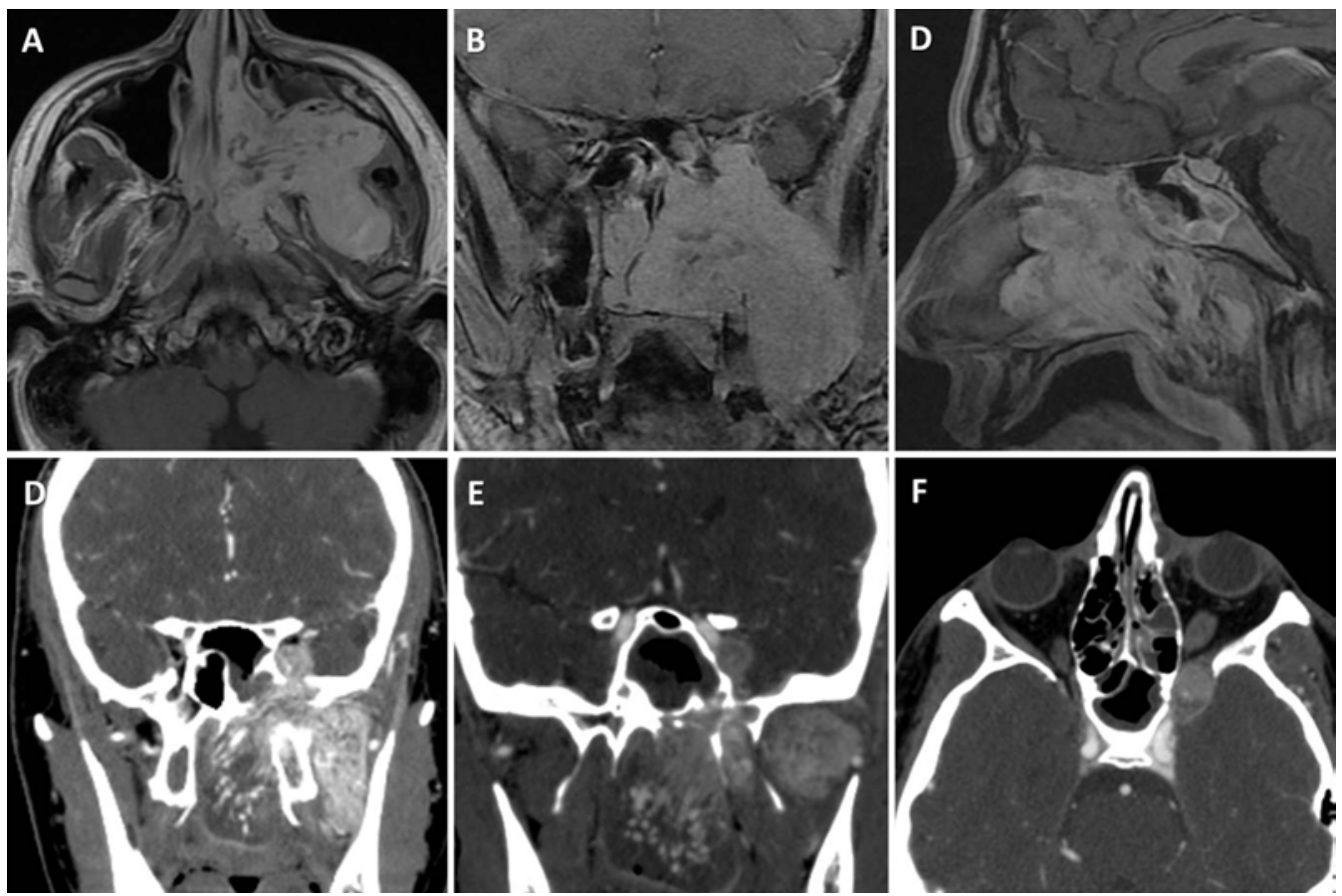

FIG. 7. Case 4. A-C: Preoperative T1 postgadolinium MRIs (axial $[\mathrm{A}]$, coronal $[\mathrm{B}]$, and sagittal views [C]) showing a large complex JNA involving the nasal cavity and left PPF with extension into the lateral ITF, buccal space, and intracranial cavity. D-F: CT angiograms (coronal [D and E], axial views [F]) show the intimate relationship of the tumor and the cavernous carotid artery.

rence. ${ }^{37}$ Stage $1-2$ tumors, according to the University of Pittsburgh Medical Center staging system, involve tumors located in the PPF, as seen in Case 1. Stage 3-4 tumors involve the ITF with skull base erosion, as seen in Cases 2 and 3. Finally, Stage 5 tumors are those with intracranial extension, as seen in Case $4 . .^{33}$
Currently, the most common approach to the ITF is the transcervical approach in a relaxed skin tension line, as it provides adequate access without poor cosmetic outcome and risk to the facial nerve. ${ }^{8}$ This approach is not without limitations, as it is particularly challenging in tumors with great medial and superior extension. ${ }^{8}$ The increased use
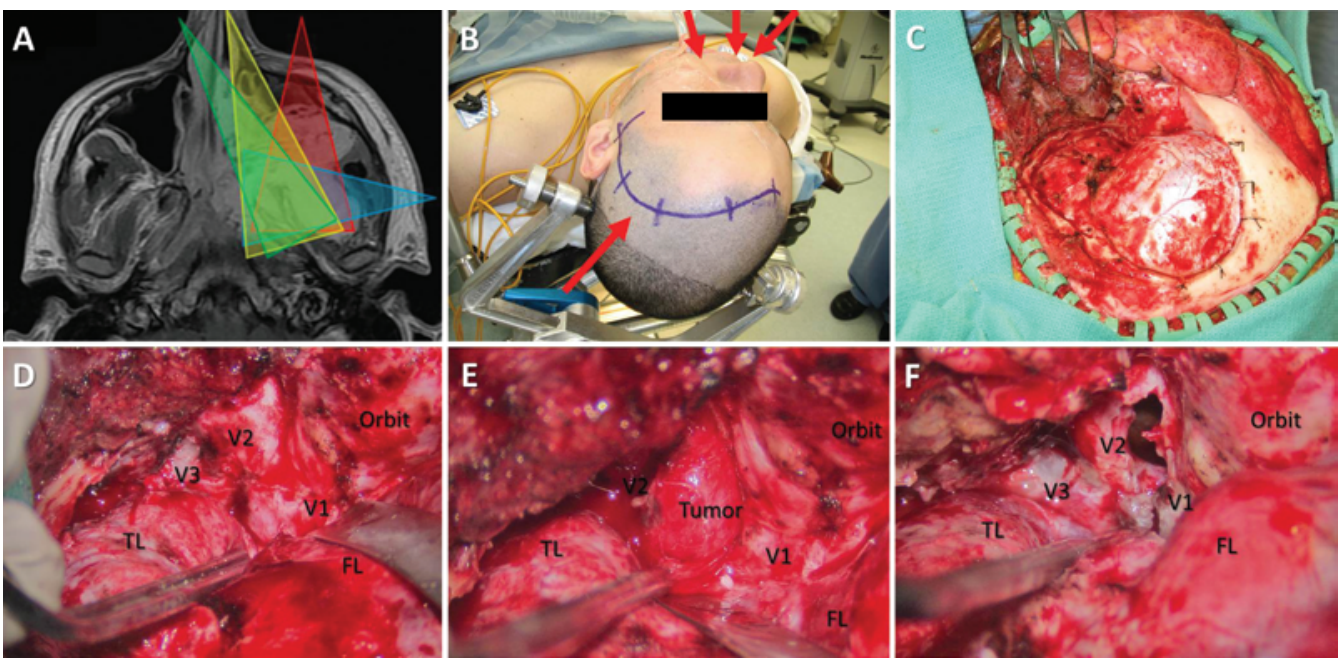

FIG. 8. Case 4. A and B: A multiangle, multicorridor approach was performed to resect the tumor using a combination of a binostril endoscopic endonasal approach, left sublabial Caldwell-Luc maxillotomy, and a left orbitozygomatic transcavernous approach. Red arrows depict the 4 corridors used in this approach. C: A left modified orbitozygomatic approach was performed. D-F: An extradural transcavernous exposure of V1 to V3 has been performed. The tumor was removed between the V1 and V2 corridor (anteromedial triangle) and vascular control of the cavernous carotid artery was obtained. $\mathrm{FL}=$ frontal lobe; $\mathrm{TL}=$ temporal lobe. V1, V2, V3 = branches of the trigeminal nerve. Figure is available in color online only. 

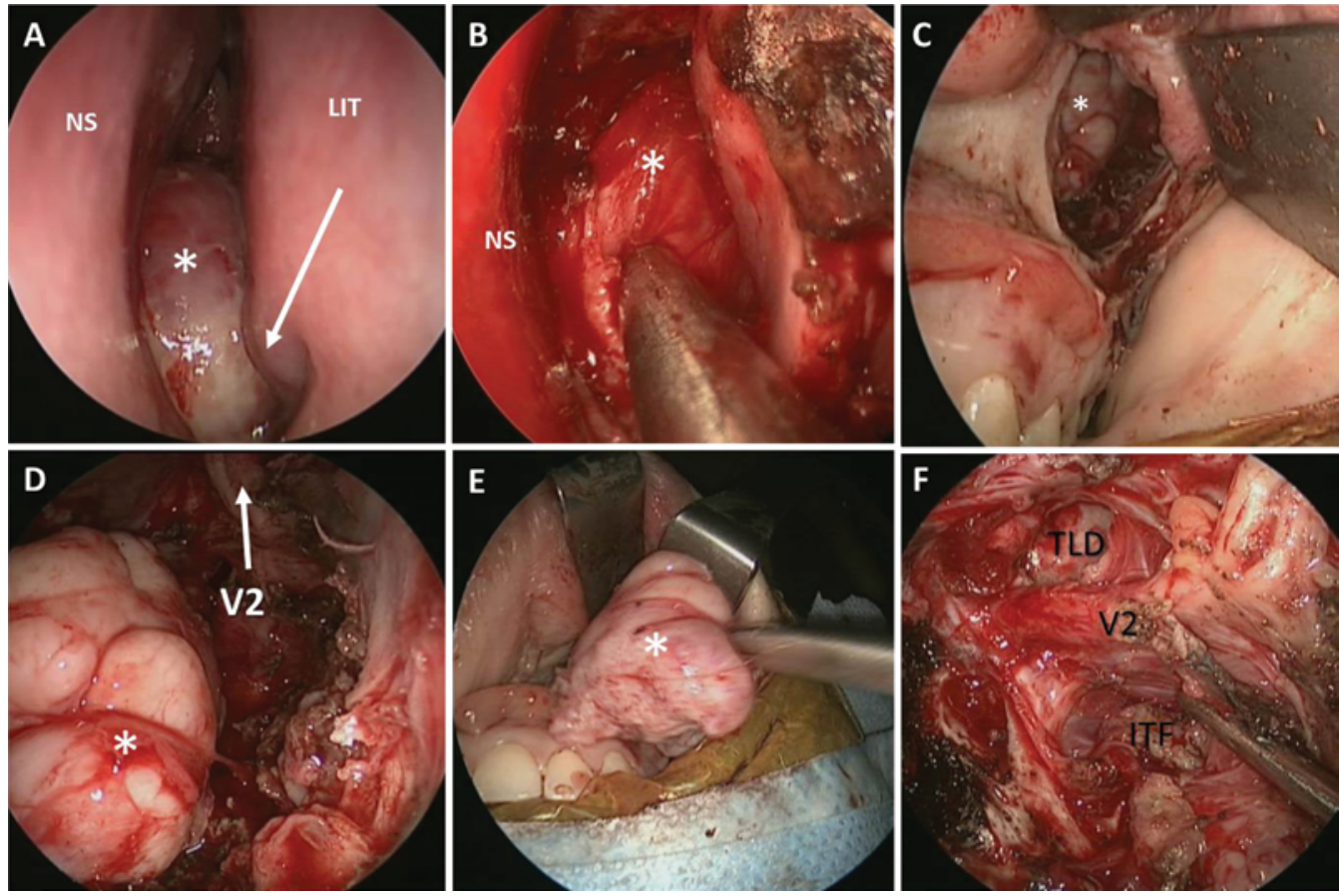

FIG. 9. Case 4. Intraoperative endoscopic views of Case 4. A: Endoscopic view of tumor (asterisk) in the nasal cavity. B: Tumor is better exposed after left medial maxillectomy. C: Visualization of tumor (asterisk) through the Caldwell-Luc maxillotomy corridor. $\mathbf{D}$ and E: Tumor in the lateral ITF resected through left Caldwell-Luc window. F: View of resection bed showing TLD, V2, and ITF. TLD = temporal lobe dura; V2 = maxillary branch of trigeminal nerve. See Figs. 2 and 4 for definitions of other abbreviations. Figure is available in color online only.

of the endoscope has led to the development of techniques that could provide increased access to challenging areas of the skull base. Other authors have described endoscopic approaches to the ITF, but many of these approaches possess a variety of limitations.

\section{Graduated Stepwise, Multiangle, Multicorridor Approach}

In our paper, we demonstrate a graduated, stepwise technique using a multiangle, multicorridor approach to these formidable skull base lesions. The 4 illustrative cases demonstrate how graduated use of multiple surgical corridors provides individualized access to JNAs involving deep areas of the skull base to facilitate effective oncologic resection (Table 1). JNAs within the nasal cavity and PPF have been well studied using the endoscopic endonasal approach.,5,9 The illumination of deep areas and panoramic visualization provided by variable angled endoscopes renders this approach far superior to open external approaches to the PPF. ${ }^{12}$

Our graduated, stepwise approach is based largely on tumor size, location, and extent (Table 2). For smaller tumors confined to the ipsilateral nasal cavity and PPF, a uninostril approach can be performed (Case 1). As JNAs increase in size and involve both nasal cavities and extend the medial aspect of the ITF, a binostril approach with a septectomy or septotomy is performed (Case 2). For tumors that extend to the lateral ITF, an additional CaldwellLuc corridor facilitates access and tumor removal from the lateral ITF (Case 3). Last, if the JNA invades intracranially, an additional transcranial corridor can be considered, particularly if vascular control of the cavernous ICA is warranted (Case 4).

In Case 1, where the tumor was located in the nasal cavity with extension to the PPF, visualization and total tumor resection were achieved using an ipsilateral (uninostril), endoscopic endonasal approach. In smaller tumors confined to this region, a purely uninostril approach can be performed to spare disruption and morbidity of the contralateral nasal cavity.

In Case 2, the tumor was larger with bilateral nasal cavity involvement and lateral extension through the PPF into the ITF. Here, a second corridor was added using the contralateral nostril and septotomy to allow for a binostril endoscopic endonasal approach using the 4-handed, 2-surgeon technique. ${ }^{17,29}$ Perfoming an ipsilateral medial maxillectomy and transpterygoid approach via binostril corridors provided more lateral access of the tumor within the ITF. ${ }^{37}$

In Case 3, the tumor involved the nasal cavity, PPF, and extended to the far lateral portion of the ITF. Thus, the addition of the third corridor (ipsilateral endoscopic Caldwell-Luc maxillotomy) provided direct access to the entire ITF. ${ }^{8,34}$ The Caldwell-Luc maxillotomy is particularly effective in lesions that are located very anterior and lateral in the region of the canine fossa ${ }^{8}$ We prefer this approach over a Denker's anteromedial maxillectomy, which may have a higher risk of cosmetic nasal deformity postoperatively. It has been previously noted, however, that there is a significant amount of nasal traction ${ }^{34}$ when performing the Caldwell-Luc procedure in tumors 

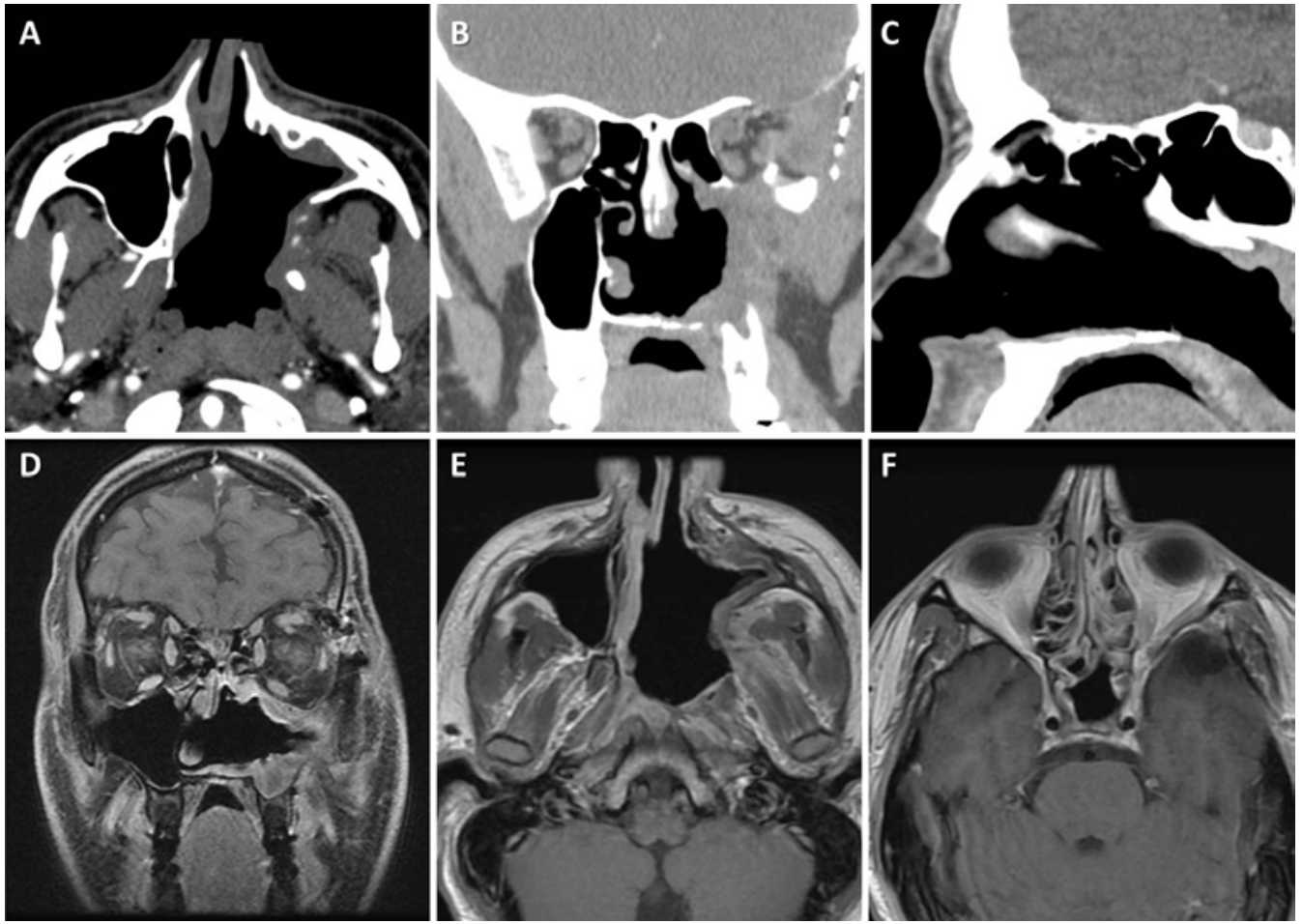

FIG. 10. Case 4. Postoperative enhanced CT and MR images. Axial (A), coronal (B), and sagittal (C) CT scans and T1-weighted post-Gd MR images (coronal [D] and axial [E and F]) at 4 months showing no evidence of residual or recurrent tumor. The cavernous sinus is free of any tumor $(F)$.

with significant posterolateral extension. ${ }^{8}$ Therefore, we have added the endoscopic contralateral transnasal septectomy approach to the endoscopically assisted ipsilateral Caldwell-Luc approach. ${ }^{8}$ The septectomy facilitates the binostril, 2-surgeon, 4-handed technique to provide greater medial access.

The patient described in Case 4 harbored the largest tumor in this series, with involvement of the lateral ITF and extension intracranially to the cavernous sinus. Here, a fourth corridor was added using an ipsilateral orbitozygomatic, extradural, transcavernous approach. In this in- stance, the orbitozygomatic approach was performed first to allow safe dissection of the tumor away from the cavernous sinus. Once the cavernous sinus and cavernous carotid were safely cleared of tumor, the remainder of the tumor was removed from below using the combined binostril and ipsilateral Caldwell-Luc maxillotomy corridors to remove the tumor from the nasal cavity and lateral ITF. Although a purely endoscopic approach from below could be considered, we felt that it was safer to do the transcranial approach first to achieve early clearance of tumor from the cavernous sinus and early vascular control of the cavern-

TABLE 2. Site-specific targets of graduated multicorridor approach

\begin{tabular}{lcc}
\hline \multicolumn{1}{c}{$\begin{array}{c}\text { Surgical } \\
\text { Corridor(s) }\end{array}$} & Approaches & Locations That Can Be Accessed \\
\hline Uninostril & $\begin{array}{c}\text { Unilat medial maxillectomy, sphenoidotomy, ethmoidectomy, } \\
\text { transpterygoid }\end{array}$ & $\begin{array}{c}\text { Ipsilat nasal cavity, sphenoid sinus, ethmoid sinus, frontal sinus, } \\
\text { hemi-skull base (cribriform), maxillary sinus, PPF, posterior } \\
\text { nasopharynx }\end{array}$ \\
\hline Binostril & $\begin{array}{c}\text { Bilat sphenoidotomy, septectomy, bilat ethmoidectomy, bilat } \\
\text { frontal sinusotomy, unilat medial maxillectomy, transptery- }\end{array}$ & $\begin{array}{c}\text { Bilat nasal cavity, sphenoid sinuses, ethmoid sinuses, frontal } \\
\text { sinuses, entire cribriform plate, ipsilat maxillary sinus, PPF, } \\
\text { posterior nasopharynx, medial ITF }\end{array}$ \\
\hline $\begin{array}{c}\text { Binostril + } \\
\text { Caldwell-Luc }\end{array}$ & $\begin{array}{c}\text { Bilat sphenoidotomy, septectomy, bilat ethmoidectomy, bilat } \\
\text { frontal sinusotomy, unilat medial maxillectomy, transptery- }\end{array}$ & $\begin{array}{c}\text { Bilat nasal cavity, sphenoid sinuses, ethmoid sinuses, frontal } \\
\text { sinuses, entire cribriform plate, ipsilat maxillary sinus, PPF, } \\
\text { posterior nasopharynx, medial and lat ITF }\end{array}$ \\
\hline $\begin{array}{c}\text { Binostril + } \\
\text { Caldwell-Luc } \\
+ \text { transcranial }\end{array}$ & $\begin{array}{c}\text { Bilat sphenoidotomy, septectomy, bilat ethmoidectomy, bilat } \\
\text { frontal sinusotomy, unilat medial maxillectomy, transptery- }\end{array}$ & $\begin{array}{c}\text { Bilat nasal cavity, sphenoid sinuses, ethmoid sinuses, frontal sinus- } \\
\text { es, entire cribriform plate, ipsilat maxillary sinus, PPF, posterior } \\
\text { goid, sublabial maxillotomy, orbitozygomatic craniotomy }\end{array}$ \\
\hline
\end{tabular}


ous carotid artery, followed by removing the remainder from the corridors from below.

In our practice, we typically operate on JNAs within 24 hours of preoperative embolization. The $30^{\circ}$ endoscope has been the workhorse for our endoscopic skull base practice, and we advance it to the $70^{\circ}$ endoscope when needed in cases that require more angled visualization. We prefer using the $30^{\circ}$ endoscope for all endoscopic skull base procedures, because of its versatility in providing the same degree of surgical exposure as the $0^{\circ}$ endoscope, but with advantages of additional angles of visualization in a multidirectional fashion without having to repeatedly exchange the 2 endoscopes. The $30^{\circ}$ endoscope, when focused laterally, is particularly helpful for JNAs that extend laterally into the ITF and maxillary sinus. Alternatively, when the endoscope is placed into the Caldwell-Luc maxillotomy corridor, the scope can be angled medially toward the maxillary antrostomy and nasal septum region. Within the nasal cavity corridors, the endoscope is angled upward to view the anterior skull base and inferiorly to better view the nasal floor and posterior nasopharynx. We typically do not use postoperative lumbar drainage when low-flow CSF leaks are encountered intraoperatively. However, selective use of postoperative lumbar drainage is considered when high-flow CSF leaks are present during surgery.

Our multiangle, multicorridor approach to JNAs of variable sizes and location represents a safe and effective strategy for surgical resection of complex JNA of the skull base. This concept of multicorridor surgery can also be applied to other neoplastic lesions, such as sinonasal malignancies and sinonasal schwannomas, involving the cranial base, particularly those involving PPF and ITF.

\section{Conclusions}

Access to complex JNA of the skull base involving multiple compartments can be gained through an endoscopic graduated multiangle, multicorridor approach. Use of a multiangled, multicorridor approach allows for greater surgical freedom and customization of access depending on the size and exact location of the tumor. Graduated use of multiple surgical corridors provides individualized access to complex JNAs involving multiple compartments within deep areas of the skull base.

\section{References}

1. Andrade NA, Pinto JA, Nóbrega MdeO, Aguiar JE, Aguiar TF, Vinhaes ES: Exclusively endoscopic surgery for juvenile nasopharyngeal angiofibroma. Otolaryngol Head Neck Surg 137:492-496, 2007

2. Andrews JC, Fisch U, Valavanis A, Aeppli U, Makek MS: The surgical management of extensive nasopharyngeal angiofibromas with the infratemporal fossa approach. Laryngoscope 99:429-437, 1989

3. Beham A, Beham-Schmid C, Regauer S, Auböck L, Stammberger H: Nasopharyngeal angiofibroma: true neoplasm or vascular malformation? Adv Anat Pathol 7:36-46, 2000

4. Boghani Z, Husain Q, Kanumuri VV, Khan MN, Sangvhi S, Liu JK, et al: Juvenile nasopharyngeal angiofibroma: a systematic review and comparison of endoscopic, endoscopicassisted, and open resection in 1047 cases. Laryngoscope 123:859-869, 2013

5. Borghei P, Baradaranfar MH, Borghei SH, Sokhandon F:
Transnasal endoscopic resection of juvenile nasopharyngeal angiofibroma without preoperative embolization. Ear Nose Throat J 85:740-743, 746, 2006

6. Chandler JR, Goulding R, Moskowitz L, Quencer RM: Nasopharyngeal angiofibromas: staging and management. Ann Otol Rhinol Laryngol 93:322-329, 1984

7. Cloutier T, Pons Y, Blancal JP, Sauvaget E, Kania R, Bresson $\mathrm{D}$, et al: Juvenile nasopharyngeal angiofibroma: does the external approach still make sense? Otolaryngol Head Neck Surg 147:958-963, 2012

8. Eloy JA, Murray KP, Friedel ME, Tessema B, Liu JK: Graduated endoscopic multiangle approach for access to the infratemporal fossa: a cadaveric study with clinical correlates. Otolaryngol Head Neck Surg 147:369-378, 2012

9. Eloy P, Watelet JB, Hatert AS, de Wispelaere J, Bertrand B: Endonasal endoscopic resection of juvenile nasopharyngeal angiofibroma. Rhinology 45:24-30, 2007

10. Enepekides DJ: Recent advances in the treatment of juvenile angiofibroma. Curr Opin Otolaryngol Head Neck Surg 12:495-499, 2004

11. Fisch U: The infratemporal fossa approach for nasopharyngeal tumors. Laryngoscope 93:36-44, 1983

12. Fyrmpas G, Konstantinidis I, Constantinidis J: Endoscopic treatment of juvenile nasopharyngeal angiofibromas: our experience and review of the literature. Eur Arch Otorhinolaryngol 269:523-529, 2012

13. Glad H, Vainer B, Buchwald C, Petersen BL, Theilgaard SA, Bonvin $\mathrm{P}$, et al: Juvenile nasopharyngeal angiofibromas in Denmark 1981-2003: diagnosis, incidence, and treatment. Acta Otolaryngol 127:292-299, 2007

14. Gullane PJ, Davidson J, O’Dwyer T, Forte V: Juvenile angiofibroma: a review of the literature and a case series report. Laryngoscope 102:928-933, 1992

15. Gupta AC, Murthy DP: Intracranial juvenile nasopharyngeal angiofibroma. Aust N Z J Surg 67:477-482, 1997

16. Hackman T, Snyderman CH, Carrau R, Vescan A, Kassam A: Juvenile nasopharyngeal angiofibroma: The expanded endonasal approach. Am J Rhinol Allergy 23:95-99, 2009

17. Harvey RJ, Sheehan PO, Debnath NI, Schlosser RJ: Transseptal approach for extended endoscopic resections of the maxilla and infratemporal fossa. Am J Rhinol Allergy 23:426-432, 2009

18. Khoueir N, Nicolas N, Rohayem Z, Haddad A, Abou Hamad $\mathrm{W}$ : Exclusive endoscopic resection of juvenile nasopharyngeal angiofibroma: a systematic review of the literature. Otolaryngol Head Neck Surg 150:350-358, 2014

19. Lloyd G, Howard D, Phelps P, Cheesman A: Juvenile angiofibroma: the lessons of 20 years of modern imaging. J Laryngol Otol 113:127-134, 1999

20. Midilli R, Karci B, Akyildiz S: Juvenile nasopharyngeal angiofibroma: analysis of 42 cases and important aspects of endoscopic approach. Int J Pediatr Otorhinolaryngol 73:401-408, 2009

21. Moulin G, Chagnaud C, Gras R, Gueguen E, Dessi P, Gaubert JY, et al: Juvenile nasopharyngeal angiofibroma: comparison of blood loss during removal in embolized group versus nonembolized group. Cardiovasc Intervent Radiol 18:158-161, 1995

22. Nicolai P, Villaret AB, Farina D, Nadeau S, Yakirevitch A, Berlucchi M, et al: Endoscopic surgery for juvenile angiofibroma: a critical review of indications after 46 cases. Am J Rhinol Allergy 24:e67-e72, 2010

23. Onerci M, Oğretmenoğlu O, Yücel T: Juvenile nasopharyngeal angiofibroma: a revised staging system. Rhinology 44:39-45, 2006

24. Onerci TM, Yücel OT, Oğretmenoğlu O: Endoscopic surgery in treatment of juvenile nasopharyngeal angiofibroma. Int $\mathbf{J}$ Pediatr Otorhinolaryngol 67:1219-1225, 2003

25. Paris J, Guelfucci B, Moulin G, Zanaret M, Triglia JM: Diag- 
nosis and treatment of juvenile nasopharyngeal angiofibroma. Eur Arch Otorhinolaryngol 258:120-124, 2001

26. Pryor SG, Moore EJ, Kasperbauer JL: Endoscopic versus traditional approaches for excision of juvenile nasopharyngeal angiofibroma. Laryngoscope 115:1201-1207, 2005

27. Radkowski D, McGill T, Healy GB, Ohlms L, Jones DT: Angiofibroma. Changes in staging and treatment. Arch Otolaryngol Head Neck Surg 122:122-129, 1996

28. Renkonen S, Hagström J, Vuola J, Niemelä M, Porras M, Kivivuori S-M, et al: The changing surgical management of juvenile nasopharyngeal angiofibroma. Eur Arch Otorhinolaryngol 268:599-607, 2011

29. Robinson S, Patel N, Wormald PJ: Endoscopic management of benign tumors extending into the infratemporal fossa: a two-surgeon transnasal approach. Laryngoscope 115:18181822,2005

30. Roger G, Tran Ba Huy P, Froehlich P, Van Den Abbeele T, Klossek JM, Serrano E, et al: Exclusively endoscopic removal of juvenile nasopharyngeal angiofibroma: trends and limits. Arch Otolaryngol Head Neck Surg 128:928-935, 2002 (Erratum in Arch Otolaryngol Head Neck Surg 129:88, 2003)

31. Scholtz AW, Appenroth E, Kammen-Jolly K, Scholtz LU, Thumfart WF: Juvenile nasopharyngeal angiofibroma: management and therapy. Laryngoscope 111:681-687, 2001

32. Sessions RB, Bryan RN, Naclerio RM, Alford BR: Radiographic staging of juvenile angiofibroma. Head Neck Surg 3:279-283, 1981

33. Snyderman CH, Pant H, Carrau RL, Gardner P: A new endoscopic staging system for angiofibromas. Arch Otolaryngol Head Neck Surg 136:588-594, 2010

34. Theodosopoulos PV, Guthikonda B, Brescia A, Keller JT, Zimmer LA: Endoscopic approach to the infratemporal fossa: anatomic study. Neurosurgery 66:196-203, 2010

35. Ye L, Zhou X, Li J, Jin J: Coblation-assisted endonasal endoscopic resection of juvenile nasopharyngeal angiofibroma. J Laryngol Otol 125:940-944, 2011
36. Yi Z, Fang Z, Lin G, Lin C, Xiao W, Li Z, et al: Nasopharyngeal angiofibroma: a concise classification system and appropriate treatment options. Am J Otolaryngol 34:133-141, 2013

37. Zanation AM, Mitchell CA, Rose AS: Endoscopic skull base techniques for juvenile nasopharyngeal angiofibroma. Otolaryngol Clin North Am 45:711-730, ix, 2012

38. Zhang M, Garvis W, Linder T, Fisch U: Update on the infratemporal fossa approaches to nasopharyngeal angiofibroma. Laryngoscope 108:1717-1723, 1998

\section{Disclosures}

The authors report no conflict of interest concerning the materials or methods used in this study or the findings specified in this paper.

\section{Author Contributions}

Conception and design: Liu, Eloy. Acquisition of data: Liu, Husain, Kanumuri, Eloy. Analysis and interpretation of data: Liu, Husain, Kanumuri, Eloy. Drafting the article: all authors. Critically revising the article: all authors. Reviewed submitted version of manuscript: all authors. Approved the final version of the manuscript on behalf of all authors: Liu. Administrative/technical/ material support: Liu, Eloy. Study supervision: Liu, Eloy.

\section{Supplemental Information \\ Videos \\ Video 1. https://vimeo.com/142900944. \\ Video 2. https://vimeo.com/142901107.}

\section{Correspondence}

James K. Liu, Department of Neurological Surgery, Rutgers New Jersey Medical School, 90 Bergen St., Ste. 8100, Newark, NJ 07103.email: james.liu.md@rutgers.edu. 